\title{
Chronic Active Hepatitis B with HBV-Associated Nephropathy: Close Resemblance to Lupus Nephritis
}

\author{
Amitesh Aggarwal ${ }^{a}$, Mukul P. Agarwal ${ }^{a}$, Surendra Rajpal ${ }^{a}$, Vineeta V. Batra ${ }^{b}$, Ankit Kumar Sahu $^{a}$ \\ ${ }^{a}$ Department of Medicine, University College of Medical Sciences \& GTB Hospital, Delhi, India \\ ${ }^{b}$ Department of Pathology, Govind Ballabh Pant hospital, New Delhi, India
}

\begin{abstract}
:
We present a rare case of a 23-year-old male incidentally detected with hepatitis B virus (HBV) infection presenting with features suggestive of HBV-associated nephropathy. A renal biopsy specimen suggested a mesangioproliferative glomerulonephritis with a full-house pattern on immunoflourescence consistent with a diagnosis of diffuse lupus nephritis. Glomerular HbeAg and $\mathrm{HbsAg}$ antigens were not detectable by immunofluorescence. Antiviral therapy was instituted to suppress viral replication, thereby leading to clinical and virological remission, including that of the glomerulonephritis, without the need for additional immunosuppressant therapy. This case depicts the uniqueness of the presentation of the two conditions mimicking each other, the strategy adopted to prevent the activation of viral replication and the achievement of clinical remission.
\end{abstract}

Keywords: Hepatitis B, HBV-associated nephropathy, lupus nephritis

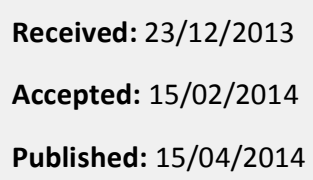




\section{Introduction}

One of the important extrahepatic manifestations of hepatitis B virus (HBV) infection is the evolution of HBV-associated nephropathy, mediated mostly by immune complex deposition. HBV-associated membranous glomerulonephritis and grade $V$ lupus membranous nephritis can at times be morphologically indistinct, with no single or combined features carrying a positive predictive value for their individual diagnosis. This is primarily because both can present as an immune complexmediated glomerulopathy with a diffuse proliferative pattern of injury and a "full-house" pattern on immunoflourescence [1]. Only the detection of viral antigens in glomeruli by the use of specific monoclonal antisera can link the virus with glomerular lesions [1]. Although as yet no clearly causal relationship has been established between HBV and lupus nephritis, this association cannot be termed merely as coincidental since immune complex deposition in lupus nephritis can be triggered by HBV. This also resulted in a dilemma in the management regarding whether antiviral or immunosuppressive therapy should be initiated first, as both disease components are critical for the long-term prognosis of the patient.

\section{Case report}

A 23-year-old male of south Asian ethnicity presented with complaints of generalised body swelling for four months, abdominal pain with progressively increasing abdominal distension for one month and low-grade fever. There was no history of oliguria, dyspnoea, chest pain, haematuria, dysuria, loss of weight or appetite, rashes, joint pain, neuropsychiatric symptoms or altered bowel habits. There was a significant past history of jaundice two years earlier that had resolved on its own in two months. There was no contributory family history of hypertension, diabetes mellitus, coronary artery disease, chronic kidney disease or similar illness.

On presentation, the patient was normotensive and mildly anaemic, with bilateral symmetrical pedal oedema. Chest examination revealed the presence of minimal bilateral pleural effusion, which was confirmed on chest X-ray.

Investigations on presentation revealed pancytopenia $(\mathrm{Hb} 9.0 \mathrm{~g} / \mathrm{dl}$, total leukocyte count $2900 / \mu \mathrm{l}$, platelet count $45,000 / \mu \mathrm{l}$ ), raised ESR of $72 \mathrm{~mm}$ in one hour, hypoalbuminaemia $1.8 \mathrm{~g} / \mathrm{dl}$ and an albumin:globulin ratio reversal (0.62). Liver function tests (LFT) initially showed mild hyperbilirubinaemia $(1.8 \mathrm{mg} / \mathrm{dl})$ with alanine transaminase (ALT) levels approximately three times above normal $(110 \mathrm{U} / \mathrm{l})$, which resolved in due course. The coagulation profile remained within normal limits (INR 1.00). Kidney function tests remained normal throughout the course of the illness. C-reactive protein (CRP) was found to be negative $(<6.0 \mathrm{mg} / \mathrm{dl}$ ), with a negative Mantoux test (03 $\mathrm{mm}$ ). $\mathrm{C}_{3}$ levels were found to be decreased to $0.34 \mathrm{~g} / \mathrm{l}$ (normal range 0.9-1.8). Anti-nuclear antibody (ANA) was found to be positive (3.8 Al) $\{N R<1\}$, though double-stranded DNA (dsDNA) was found to 
be negative. HIV and anti-HCV were negative.

The patient was put on enalapril ( $5 \mathrm{mg} /$ day) and furosemide (40 $\mathrm{mg}$ twice a day). Hepatitis $B$ surface antigen ( $\mathrm{HbsAg}$ ) was found to be positive.

HBV DNA titre was $1 \times 10^{6}$ copies/ml, hepatitis-B ' $\mathrm{e}$ ' antigen ( $\mathrm{HbeAg}$ ) was positive, and anti-HbeAg antibody was negative. Urine evaluation showed moderate proteinuria (24-h urinary protein was $1872 \mathrm{mg})$.

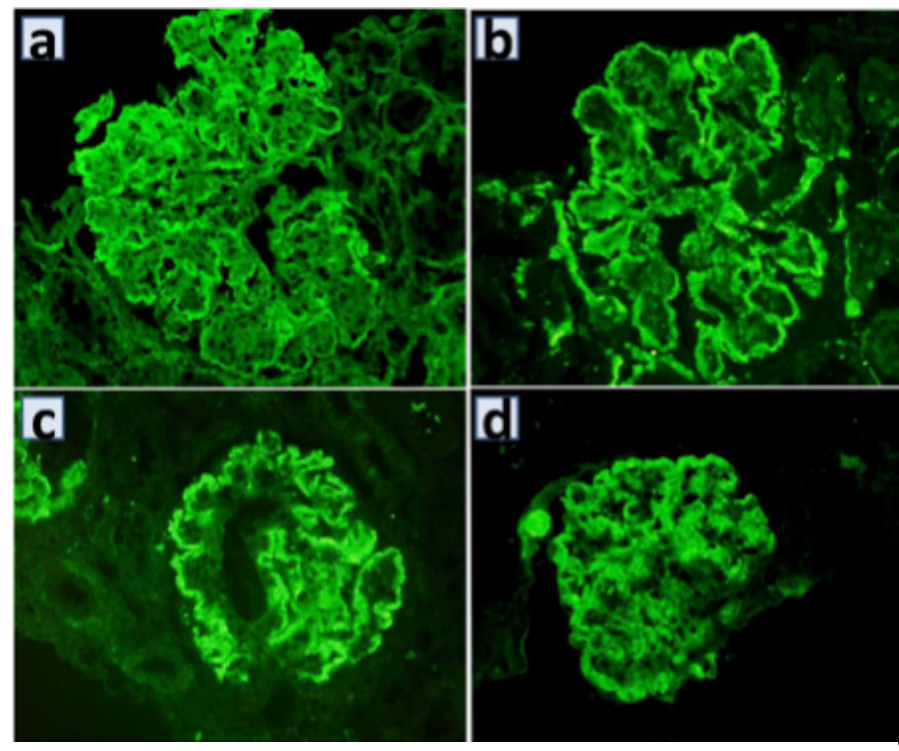

Figure 1: Light microscopy photomicrographs. $(a, b)$ Haematoxylin and eosin (HE)-stained sections of kidney biopsy at low- and high-power view showing enlarged glomeruli displaying an increase in mesangial matrix and cellularity (magnification 100x and 200x, respectively).(c,d) Periodic acid Schiff (PAS)- and silver methenamine (Sm)-stained sections of kidney biopsy at high power displaying splitting of basement membranes (magnification 400x).

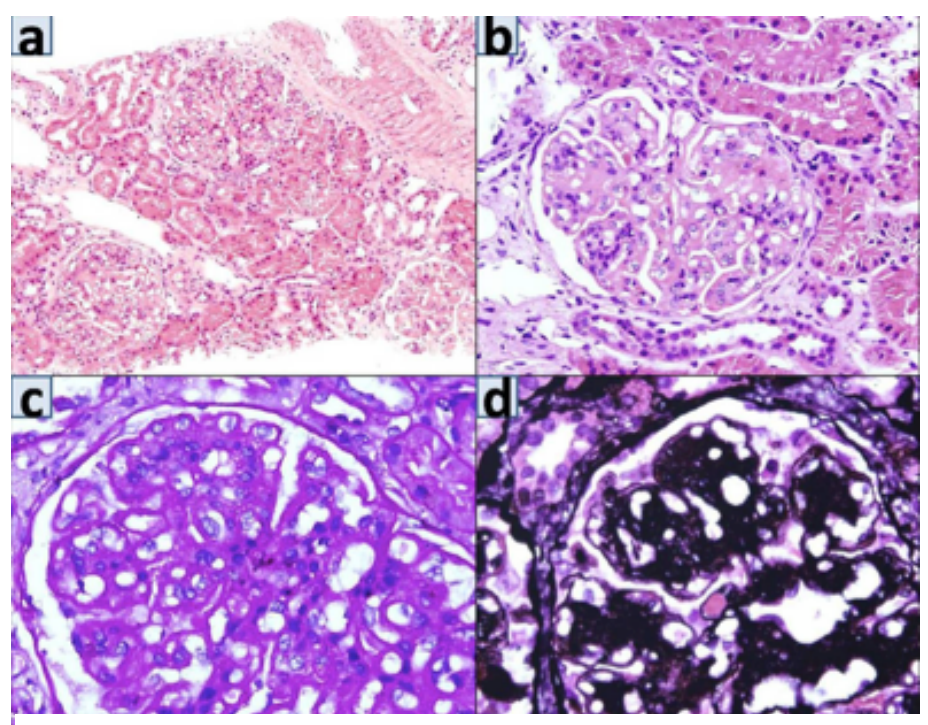

Figure 2: Immunofluorescence photomicrographs. (a-d) Frozen sections of kidney biopsy stained with $\lg G, C 3, \lg M$ and $C 1 q$, respectively, displaying immune complex and complement deposition along the peripheral capillary walls and mesangium as fine granular deposits (magnification 200x).
Abdominal ultrasound showed coarse liver echo texture, splenomegaly with multiple portosystemic collaterals and normal-sized kidneys with well maintained corticomedullary differentiation.

On renal biopsy (Fig. 1a-d), immunoflourescence showed a 'full-house' pattern with deposition of immunoglobulin $G, M, A \quad(\operatorname{IgG})$ and complements $\mathrm{C}_{3}$ and C1q. (Fig. 2a-d).

In view of the serological investigations and immunofluorescence findings, a diagnosis of diffuse lupus nephritis Class IV (ISN/RPS classification) was made. Glomerular deposition of $\mathrm{HbeAg}$ and HbsAg could not be demonstrated by immunohistochemistry. The patient's anasarca responded to medical therapy. Immunosuppressants were withheld for fear of hepatic decompensation due to flaring up of hepatitis B infection.

The patient was subsequently put on 0.5 $\mathrm{mg}$ of entecavir $0.5 \mathrm{mg}$ once daily. The patient is currently in clinical and virological sustained remission (HBV DNA titre: 100 copies $/ \mathrm{ml}$ ). Following the antiviral therapy, the proteinuria remarkably reduced to $200 \mathrm{mg} /$ day. A clinical diagnosis of chronic active hepatitis B with HBV-associated

:an Journal of Case Reports in Internal Medicine (C) EFIM 2014 
glomerulonephritis was made.

\section{Discussion}

The uniqueness of our case was the initial discovery of chronic active hepatitis B along with nephritis histologically resembling diffuse lupus nephritis despite normal kidney function tests and the absence of clinical hepatic deterioration.

Impaired production of interferon in peripheral blood mononuclear cells has been reported to be potentially pathogenic to both chronic HBV infection and systemic lupus erythematosus (SLE). The immunoflourescence examination shows that although the deposition of $C_{3}$ and $C_{1 q}$ is more frequent in HBV membranous glomerulonephritis as compared to membranous glomerulonephritis due to other secondary causes, a 'full-house' pattern is not very common [2]. Previous experiments have suggested that initiation of immune-tolerant therapy in the form of interferon- $\alpha$ has precipitated severe SLE forms in patients being treated for HCV or HBV with or without coexisting HIV infection [3]. Therefore, immunosuppressant therapy for lupus risks exacerbating underlying infection in patients with concurrent HIV, HBV or HCV by reactivating the viral replication in the presence of decreased immunity, which can be very detrimental [4].

There is some uncertainty as to how to manage nephritis and chronic active hepatitis B simultaneously, but our approach of targeting the hepatitis first seems to have been very effective. Corticosteroids given at the onset of nephrotic syndrome in HBV membranous nephropathy (HBVMN) do not seem to have an ameliorative effect on the nephrotic state or viral clearance [5]. On the contrary, serological evidence of active viral replication and histological deterioration did not support the protective value of steroids in HBVMN. More recently, Tse et al. recommended lamivudine treatment for virological flares in managing chronic hepatitis B patients with a history of lupus nephritis [6]. We therefore initiated antiviral therapy in the form of entecavir to achieve virological remission instead of immunosuppressive therapy for the management of lupus nephritis.

\section{Conlcusion}

This case report illustrates the complex interactions between HBV, SLE and antiviral immunity. The risks of viral reactivation after initiation of immunosuppressive therapy for treatment of autoimmune diseases like SLE need careful assessment and monitoring. Immunosuppressant therapy is a double-edged sword in this setting and therefore its benefits need to be balanced against its hazards. Therefore, in the absence of detection of hepatitis antigens in glomeruli, HBV-associated glomerulonephritis can be confused with lupus nephritis. 


\section{Learning Points}

- HBV-associated membranous glomerulonephritis and grade $V$ lupus membranous nephritis can at times be morphologically indistinct with no single or combined features carrying a positive predictive value for their individual diagnosis.

- There is uncertainty in management as to whether to first initiate antiviral or immunosuppressive therapy, as both the disease components are critical for the long-term prognosis of the patient.

- It is currently recommended to initiate antiviral therapy for hepatitis to achieve virological remission instead of immunosuppressive therapy for the management of lupus nephritis for virological flares in chronic hepatitis B patients with a history of lupus nephritis.

\section{References}

1. Lai FM, To KF, Wang AYM, Choi PCL, Szeto CC, Li PKT et al. Hepatitis B virus-related nephropathy and lupus nephritis: morphologic similarities of two clinical entities, Mod Pathol 2000;13:166-172.

2. Lai KN, Lai FM, Chan KW, Chow CB, Tong KL, Vallance-Owen J. The clinico-pathologic features of hepatitis B virus-associated glomerulonephritis, Q J Med 1987;63:323-333.

3. Ho V, Mclean A, Terry S. Severe systemic lupus erythematosus induced by antiviral treatment for hepatitis C, J Clin Rheumatol 2008;14:166-168.

4. Abbott IJ, Chang CC, Skinner MJ, Street A, Perry G, McLean C et al. Development and management of systemic lupus erythematosus in an HIV-infected man with hepatitis $C$ and $B$ co-infection following interferon therapy: a case report, J Med Case Rep 2009;3:7289.

5. Bhimma R, Coovadia HM. Hepatitis B virus-associated nephropathy, Am J Nephrol 2004;24:198-211.

6. Lai FM, Lai KN, Lee JC, Hom BL. Hepatitis B virus-related glomerulopathy in patients with systemic lupus erythematosus, Am J Clin Pathol 1987;88:412-420. 